\title{
Análise do comportamento mecânico das ligas AA7004 e AA7075 tixoforjadas em prensa excêntrica
}

Luis Vanderlei Torres ${ }^{1,2 *}$ Eugênio José Zoqui ${ }^{2}$

\section{Resumo}

A tecnologia de processamento de ligas metálicas no estado semissólido tem evoluído muito nos últimos anos, devido a inúmeras vantagens em comparação com os processos convencionais de manufatura, a saber: maior eficiência energética, maior produtividade, obtenção de microestruturas refinadas, boas propriedades mecânicas, melhoria da qualidade dos produtos, entre outras. Sendo assim, este trabalho visa analisar o comportamento mecânico em tração das ligas AA7004 e AA7075 produzidas via tixoforjamento; as ligas foram reaquecidas até as temperaturas correspondentes à fração líquida de $55 \%$ e mantidas pelo tempo de 210 segundos e na sequência tixoforjadas em uma prensa excêntrica. As ligas apresentaram morfologia globular antes do processo de tixoforjamento e imediatamente após o processo de tixoforjamento apresentaram grãos totalmente deformados devido à deformação sofrida durante o processo, obtendo valores satisfatórios de limite de resistência à tração demonstrando que ambas as ligas são propícias ao processamento semissólido.

Palavras-chave: Material semissólido; Tixoforjamento; Comportamento mecânico; AA7004; AA7075.

\section{Analysis of mechanical behavior of AA7004 and AA7075 alloys thixoforging in an eccentric press}

\begin{abstract}
The technology of metal alloys processing in the semisolid state has evolved greatly in recent years, due to numerous advantages compared to conventional manufacturing processes, namely: higher energy efficiency, higher productivity, obtaining refined microstructures, good mechanical properties, product quality improvement, among others. Thus, this work aims to analyze the mechanical behavior of AA7004 and AA7075 alloys produced by thixoforging process; the alloys were reheated to temperatures corresponding to the $55 \%$ liquid fraction and maintained for the time of 210 seconds and in the thixoforged sequence for an eccentric press. The alloys showed globular morphology before the thixoforging process and immediately after the thixoforging process presented grains totally deformed due to the deformation suffered during the process, obtaining satisfactory values of tensile strength, demonstrating that both alloys are suitable for semisolid processing.
\end{abstract}

Keywords: Semisolid material; Thixoforging; Mechanical behavior; AA7004; AA7075.

\section{Introdução}

O processamento de materiais no estado semissólido possui duas vertentes, reofundição e tixoconformação. Enquanto o processo de reofundição refere-se à obtenção de peças a partir do estado líquido, o processo de tixoconformação faz o caminho inverso utilizando matéria-prima inicialmente no estado sólido. Para realizar o processo de tixoconformação com êxito necessita-se compreender a influência de algumas variáveis importantes, tais como: composição química (que define o intervalo da temperatura de trabalho), morfologia da matéria-prima (deve possuir uma morfologia globular), temperatura de trabalho (temperatura de reaquecimento do material no estágio semissólido) e tempo de permanência do material no estado semissólido (tempos pequenos para evitar o crescimento excessivo dos grãos) [1-5].

O alumínio, apesar de ser o terceiro elemento mais abundante na crosta terrestre é o metal mais jovem usado em

${ }^{1}$ Instituto Federal de Educação, Ciência e Tecnologia de São Paulo - IFSP, Bragança Paulista, SP, Brasil.

${ }^{2}$ Departamento de Engenharia de Manufatura e Materiais, Faculdade de Engenharia Mecânica, Universidade Estadual de Campinas - UNICAMP, Campinas, SP, Brasil.

*Autor correspondente: torres@ifsp.edu.br 
escala industrial, sendo produzido comercialmente há cerca de 150 anos. Sua produção atual supera a soma de todos os outros metais não ferrosos [6]. O rápido crescimento do alumínio na indústria é resultado de uma série de fatores: apresentam excelentes combinações de propriedades mecânicas, boa fundibilidade, baixa densidade (aproximadamente um terço da densidade do aço) o que somado à sua elevada resistência mecânica torna-se viável à indústria de manufatura [7]. As ligas de alumínio da série 7XXX encontram sua principal aplicação na indústria aeronáutica, uma vez que essas ligas atingem níveis elevados de resistência mecânica entre as ligas de alumínio [8] fator que tem despertado interessante à tecnologia de processamento de ligas metálicas no estado semissólido. Portanto, este trabalho tem como objetivo apresentar resultados sobre a viabilidade de se utilizar as ligas AA7004 e AA7075 como matéria-prima para os processos de tixoconformação, sendo estas ligas tixoforjadas em prensa excêntrica e na sequência submetidas a ensaios de tração para a caracterização de suas propriedades mecânicas.

\section{Materiais e métodos}

Seguindo a metodologia adotada em trabalhos anteriores, ou seja, caracterização da temperatura de trabalho, ensaios de tixoforjamento, caracterização microestrutural e caracterização do comportamento mecânico [9-11], este trabalho tem como objetivo avaliar a tixoconformabilidade das ligas de alumínio da série 7XXX através de ensaios de tixoforjamento em prensa excêntrica utilizando matriz fechada em termos de comportamento mecânico do produto final.

\subsection{Matéria-prima e caracterização da temperatura de trabalho}

As matérias-primas utilizadas para o desenvolvimento deste trabalho foram as ligas comerciais de alumínio AA7004 eAA7075 produzidas via lingotamento contínuo convencional pela empresa ALCOAAlumínio S.A, sendo suas composições químicas em peso ( $\mathrm{wt} \%$ ) apresentadas na Tabela 1 conforme fabricante. As temperaturas de trabalho foram determinadas via ensaios de análise térmica de calorimetria diferencial de varredura (DSC) através do equipamento de análise térmica NETZSCH modelo STA 409C com uma taxa de aquecimento de $5^{\circ} \mathrm{C} / \mathrm{min}$ até a temperatura de $700{ }^{\circ} \mathrm{C}$ e resfriamento até temperatura ambiente; sendo assim, com os dados obtidos é possível construir uma curva de fração líquida versus temperatura para cada liga, utilizando como base os modelos da Regra da Alavanca e Scheil, o que fornece diretamente as temperaturas (pontuais) de trabalho correspondentes à fração líquida de 55\%, conforme Tabela 1 .

\subsection{Ensaios de tixoforjamento}

A matriz de tixoforjamento utilizada neste trabalho foi projetada através da ferramenta gráfica software Pro Engineer com o intuito de obter um produto final que atendesse alguns critérios, a saber: possuir geometria suficiente para a retirada de dois corpos de prova para o ensaio de tração, área central do corpo de prova deveria possuir a maior deformação durante o processo de tixoforjamento e possuir raios e chanfros de maneira a facilitar o escoamento do material dentro da matriz [10]; a geometria do produto final (cor preta) com a respectiva geometria do corpo de prova de tração (cor vermelha) e a matriz de tixoforjamento podem ser vistas nas Figuras 1a e 1b.

As ligas de alumínio foram tixoforjadas em uma prensa excêntrica com capacidade de 25 toneladas e instrumentada. O procedimento estabelecido para a realização do ensaio de tixoforjamento consistiu no aquecimento dos corpos de prova (lingotes) com dimensões de $145 \mathrm{~mm}$ de comprimento e $27,5 \mathrm{~mm}$ de diâmetro e com furo de $1,6 \mathrm{~mm}$ de diâmetro na metade de seu diâmetro para ser inserido o termopar com suas respectivas temperaturas de trabalho, bem como no aquecimento por uma resistência elétrica (aproximadamente $200{ }^{\circ} \mathrm{C}$ ) da matriz confeccionada com aço AISI H13 (com dureza aproximada de $48 \mathrm{HRC}$ ). Os corpos de prova (lingotes) eram inseridos no forno resistivo com um termopar tipo K, Cromel Alumel, fixado em seu interior. Após alcançar a temperatura relativa à fração líquida de 55\% e o tempo de reaquecimento de 210 segundos, o corpo de prova (lingote) era imediatamente transferido para a matriz, retirando-se o termopar de sua extremidade (evitando ao máximo a perda de temperatura). Posicionado o corpo de prova dentro da matriz a prensa era acionada; com força máxima de fechamento da matriz de aproximadamente $500 \mathrm{~kg}$ para ambas as ligas [12]; sendo realizadas três réplicas para cada liga, totalizando 6 corpos de prova para o ensaio de tração. Os produtos tixoforjados foram submetidos ao processo de corte em uma serra mecânica na metade de sua largura, posteriormente, as duas metades foram usinadas para a obtenção de corpos de prova para os ensaios de tração, regido pela norma ASTM B557M-02a [15], conforme Figura 1a. Ressalta-se que a condição escolhida para a realização dos experimentos, ou seja, 55\% de fração líquida e tempo de reaquecimento de 210 segundos devem-se aos baixos valores de viscosidade aparente apresentados durante ensaios de compressão a quente [13].

Tabela 1. Composição química em peso (wt\%) das ligas AA7004 e AA7075 conforme fabricante e temperaturas de trabalho obtidas através de ensaios de DSC $[12,13]$

\begin{tabular}{ccccccccc}
\hline Liga & $\mathbf{Z n}$ & $\mathbf{M g}$ & $\mathbf{C u}$ & $\mathbf{F e}$ & $\mathbf{S i}$ & $\mathbf{M n}$ & Al & Temp. trabalho [ $\left.{ }^{\circ} \mathbf{C}\right]$ \\
\hline AA7004 & 5,8 & 1,5 & 0,1 & 0,4 & 0,3 & 0,5 & Balanço & 647 \\
AA7075 & 6,2 & 2,0 & 1,7 & 0,5 & 0,4 & 0,1 & Balanço & 614 \\
\hline
\end{tabular}



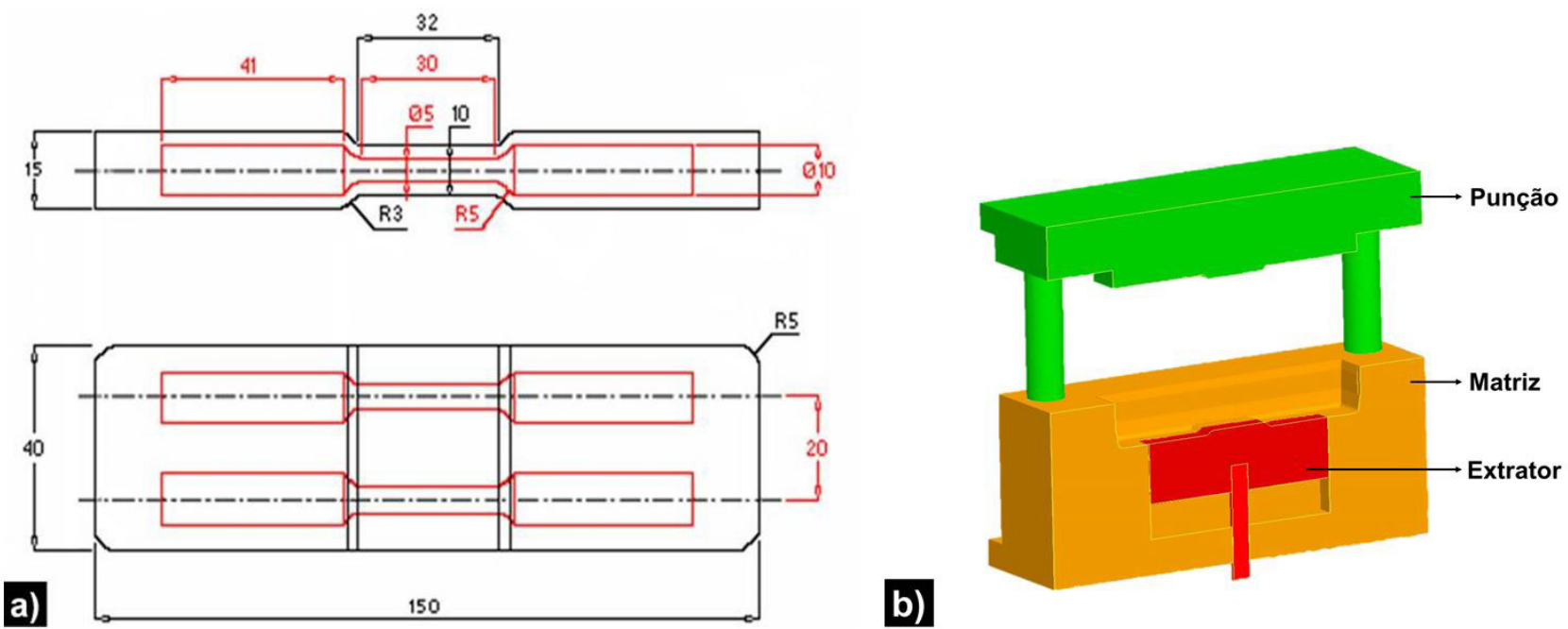

Figura 1. Dimensões do produto tixoforjado (cor preta) e dimensões dos corpos de prova para o ensaio de tração (cor vermelha) adaptado de Torres e Zoqui [12] e Nadal et al. [14] (a) e a vista em corte da matriz projetada e utilizada no processo de tixoforjamento (b).

Após os ensaios, foram realizadas análises microestruturais via metalografia convencional, sendo lixadas em lixa d'água de $220,320,400,600,800,1200$ e 1500 e polidas com pasta de diamante de $6 \mu \mathrm{m}$ (polimento grosseiro) e $1 \mu \mathrm{m}$ (polimento de acabamento). Após o polimento, as amostras foram atacadas com reagente $\operatorname{Keller}\left(2,5 \mathrm{~mL} \mathrm{HNO}_{3}, 1,5 \mathrm{~mL}\right.$ $\mathrm{HCl}, 1 \mathrm{~mL} \mathrm{HF}$ e $95 \mathrm{~mL} \mathrm{H}_{2} \mathrm{O}$ ) pelo tempo de 10 segundos, sendo utilizado para a aquisição das imagens um microscópio óptico Leica DM ILM. As mesmas amostras passaram pela caracterização microestrutural via metalografia colorida através de um ataque eletrolítico com deposição de $\mathrm{HBF}_{4}$ (ácido fluorbórico) em solução $6,0 \%$ e tensão de $20 \mathrm{~V}$ por cerca de 90 segundos com agitação moderada e constante $[10,12,13]$. As medições de tamanho de glóbulos primários e de grãos foram obtidas pelo Método dos Interceptos de Heyn, regido pela norma ASTM E112 [16].

\subsection{Ensaios de tração}

Os ensaios de tração têm como objetivo determinar as propriedades mecânicas de tensão limite de escoamento, alongamento e limite de resistência à tração. Os ensaios foram realizados em uma máquina universal de ensaios MTS, modelo 810 , sendo utilizado para o monitoramento da carga uma célula de carga com capacidade de $100 \mathrm{kN}$ e velocidade de ensaio de $0,02 \mathrm{~mm} / \mathrm{s}$; sendo a célula de carga ligada ao sistema Test Star II, mediante o aplicativo Testware $S X$. Os dados de saída da máquina universal de ensaios foram força, deslocamento e tempo para cada instante do ensaio; sendo utilizadas três réplicas para cada condição.

\section{Resultados e discussões}

Nesta seção serão apresentados e discutidos os resultados obtidos, conforme descrito na seção anterior. Para tanto, os resultados são apresentados segundo a ordem em que foram mencionados, buscando facilitar a compreensão e discussão dos resultados. Assim, os produtos tixoforjados das ligas em estudo apresentaram total preenchimento da matriz devido à utilização da fração líquida de $55 \%$, ou seja, apresentando maior quantidade de fase líquida no material, contribuindo para uma melhor fluidez e consequentemente uma boa conformabilidade do produto final.

Para uma melhor compreensão do que ocorre com a microestrutura das ligas após o processo de tixoforjamento foram realizadas análises microestruturais para a visualização dos glóbulos primários e dos grãos. O tamanho de grão na microestrutura é fácil de reconhecer, contrariamente ao tamanho de glóbulo primário, assim, neste trabalho convencionou-se denominar como grão à unidade presente na metalografia colorida (partículas com a mesma coloração) e como glóbulo primário a partícula primária existente na metalografia convencional. Ressalta-se que para estas análises foram retiradas amostras da área central, uma vez que a geometria do produto lhe conferia esse resultado, pois tal deformação ocorre justamente na área que sofreria a maior solicitação mecânica, ou seja, a área útil do corpo de prova; também foram analisadas as amostras antes do processo de tixoforjamento, para efeito de comparação microestrutural.

Analisando as microestruturas das ligas antes do processo de tixoforjamento, \& s $2 \mathrm{a}$ e $2 \mathrm{~b}$ e $3 \mathrm{a}$ e $3 \mathrm{~b}$, nota-se uma morfologia globular para a liga AA7075 e uma morfologia praticamente globular para a liga AA7004, sendo que ambos os fenômenos de ostwald ripening (mecanismo de engrossamento dos ramos dendríticos) e coalescência agiram simultaneamente para a obtenção desta microestrutura, demonstrando dependência em relação ao tempo de permanência da liga à temperatura de tratamento térmico [17,18]; outro fato observado foi a diminuição desses valores quando a liga passa pelo processo de tixoforjamento principalmente quando analisamos os valores médios de grãos, devido à deformação 

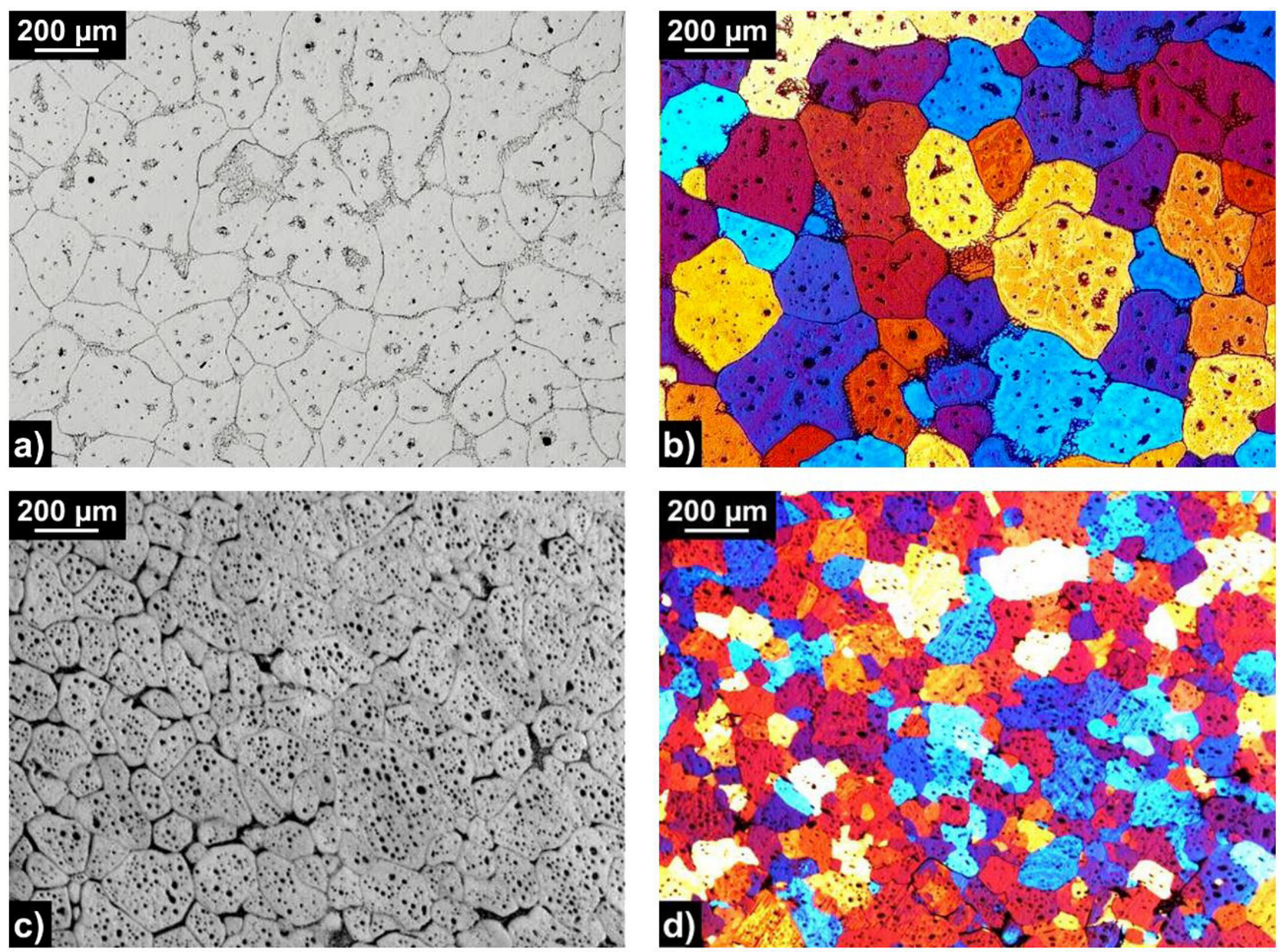

Figura 2. Comparação microestrutural da liga AA7004 para a condição de 55\% de fração líquida e tempo de tratamento de 210 segundos: antes do processo de tixoforjamento $(a, b)$ e após o processo de tixoforjamento $(c, d)$.

sofrida durante o processo, tornando os glóbulos primários e grãos totalmente deformados, Figuras $2 \mathrm{c}$ e $2 \mathrm{~d}$ e $3 \mathrm{c}$ e $3 \mathrm{~d}$.

Como observado em trabalho anterior [12] a liga AA7075 apresentou o tamanho de glóbulo primário superior quando comparada com a mesma liga antes do processo de tixoforjamento, este fato pode ser explicado devido à deformação sofrida, fazendo com que os contornos de grãos praticamente desaparecessem. A Tabela 2 apresenta os valores médios de tamanho de glóbulos primários e de grãos.

Após a obtenção dos produtos tixoforjados, estes foram usinados para a retirada dos corpos de prova de tração; a geometria e as dimensões adotadas para confecção dos corpos de prova de tração encontram-se na Figura 1a. O objetivo de tal ensaio é a determinação das propriedades mecânicas do material, assim temos: (a) limite de escoamento que consiste na tensão em que o material começa a deformar-se plasticamente sendo para o alumínio de $0,2 \%$ do comprimento original do corpo de prova, (b) alongamento que indica a ductilidade do material e (c) limite de resistência à tração que corresponde à tensão máxima que o material pode suportar antes de sua ruptura, sendo que toda a deformação até este ponto é uniforme ao longo da região do corpo de prova que se encontra sob tração, contudo, com esta tensão máxima, começa-se a formar um pequeno "estrangulamento" em algum ponto determinado e toda a deformação fica confinada a esta região [19].

Na Tabela 3, têm-se as propriedades mecânicas obtidas nos ensaios de tração, observa-se que o limite de resistência à tração de ambas as ligas ficou próximos a $200 \mathrm{MPa}$, ou seja, resultados promissores quanto à utilização das mesmas como matéria-prima para os processos de tixoconformação.

As ligas alumínio-silício (A356 e A357) são as mais utilizadas nos processos que envolvem materiais semissólidos ocupando $95 \%$ de todas as aplicações, enquanto que todas as outras ligas representam somente $5 \%$ [20], desta forma, é essencial o estudo de outras ligas para o uso nos processos de tixoconformação e este trabalho visa justamente colaborar com o desenvolvimento da utilização das ligas semissólidas AA7004 e AA7075. Torres e Zoqui [21] obteve valores de dureza vickers muito superiores quando comparado com a condição fundida, a saber, para a liga AA7004 um aumento de $75 \mathrm{HV}$ e para a liga AA7075 um aumento de $85 \mathrm{HV}$, devido às mesmas terem sido tixoconformadas. Zoqui et al. [9] 

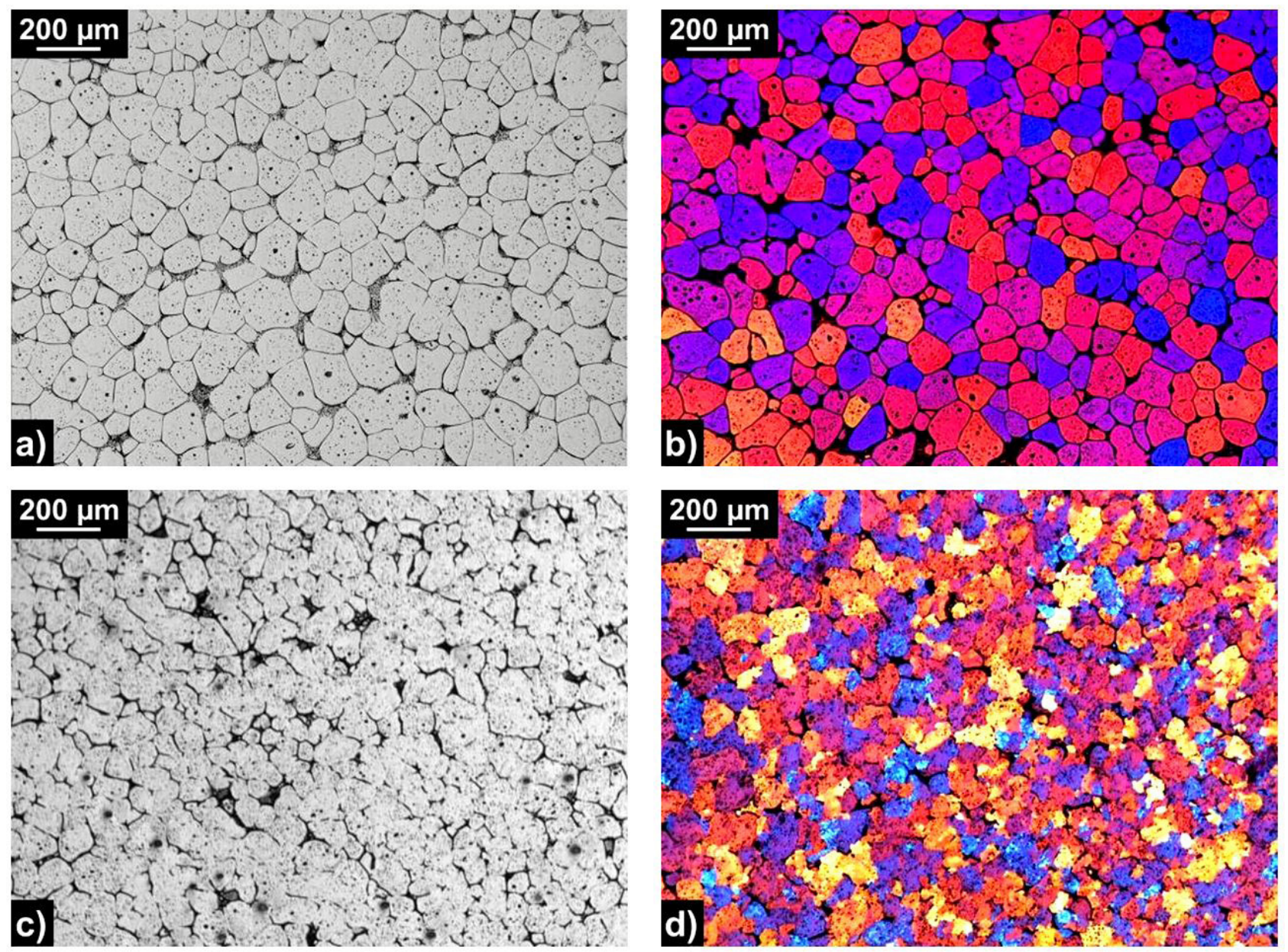

Figura 3. Comparação microestrutural da liga AA7075 para a condição de 55\% de fração líquida e tempo de tratamento de 210 segundos: antes do processo de tixoforjamento $(a, b)$ e após o processo de tixoforjamento $(c, d)$.

Tabela 2. Caracterização microestrutural das ligas: tamanho médio de glóbulo primário e tamanho médio de grão antes do processo de tixoforjamento [13] e após o processo de tixoforjamento [12]

\begin{tabular}{|c|c|c|c|}
\hline Liga & Condição & Glóbulo primário $[\mu \mathrm{m}]$ & Grão $[\mu \mathrm{m}]$ \\
\hline \multirow{2}{*}{ AA7004 } & Antes do tixoforjamento & $189 \pm 18$ & $307 \pm 60$ \\
\hline & Após o tixoforjamento & $129 \pm 31$ & $142 \pm 12$ \\
\hline \multirow{2}{*}{ AA7075 } & Antes do tixoforjamento & $76 \pm 5$ & $137 \pm 32$ \\
\hline & Após o tixoforjamento & $128 \pm 22$ & $105 \pm 24$ \\
\hline
\end{tabular}

Tabela 3. Valores de tensão limite de escoamento, alongamento e limite de resistência à tração das ligas estudadas

\begin{tabular}{cccc}
\hline Liga & Tensão limite de escoamento (MPa) & Alongamento (\%) & $\begin{array}{c}\text { Limite de resistência à tração } \\
(\mathbf{M P a})\end{array}$ \\
\hline AA7004 & $115,3 \pm 13$ & $4,4 \pm 1,6$ & $194,2 \pm 19$ \\
AA7075 & $166,2 \pm 18$ & $<1,0 \pm 0,01$ & $188,9 \pm 17$ \\
\hline
\end{tabular}

utilizando o mesmo processo de tixoforjamento em prensa excêntrica com várias ligas Al-Si obteve valores inferiores de comportamento mecânico em tração quando comparados com as ligas deste estudo. Vilas Bôas [22] também utilizando o mesmo processo de tixoforjamento em prensa excêntrica com três diferentes ligas Al-Si-Zn obteve valores inferiores de limite de escoamento e de limite de resistência à tração. Chayong et al. [23] analisaram a liga AA7075 sob a condição extrudada conseguindo valores de limite de resistência à tração de $235 \mathrm{MPa}$ após o processo de tixoconformação; comparando o valor obtido com o presente estudo, tem-se um aumento de $46 \mathrm{MPa}$, porém isto deve-se ao fato da liga estar sob a condição extrudada e durante seu reaquecimento à temperatura semissólida houve a recristalização do material, proporcionando uma microestrutura mais homogênea e consequentemente um aumento na propriedade mecânica. Jiang et al. [24] obtiveram valores de limite de resistência à tração de aproximadamente $200 \mathrm{MPa}$ para a liga AA7005 sob 
a condição extrudada e T6 e na sequência tixoconformada (composição química próxima da liga AA7004), ou seja, apenas $6 \mathrm{MPa}$ a mais quando comparado com os resultados deste trabalho. Assim, tem-se que os valores obtidos neste trabalho são satisfatórios, uma vez, que as ligas utilizadas se encontravam na condição apenas fundida.

\section{Conclusões}

Este trabalho apresenta as seguintes conclusões quanto à análise sobre o comportamento mecânico em tração das ligas AA7004 e AA7075 tixoforjadas em prensa excêntrica: (a) bom comportamento microestrutural, ou seja, ambas as ligas apresentaram morfologia globular e após o processo de tixoforjamento apresentaram a diminuição do tamanho de grão, devido à deformação sofrida durante o processo e (b) bom comportamento mecânico, com limites de resistência à tração próximos a $200 \mathrm{MPa}$, ou seja, resultados promissores quanto à utilização das mesmas como matéria-prima para os processos de tixoconformação.

\section{Agradecimentos}

Os autores agradecem a empresa ALCOAAlumínio S.A. pela doação da matéria-prima e a CAPES (Coordenação de Aperfeiçoamento de Pessoal de Nível Superior) por seu apoio financeiro. Agradecemos também à FEM/UNICAMP Faculdade de Engenharia Mecânica da Universidade Estadual de Campinas e ao IFSP - Instituto Federal de Educação, Ciência e Tecnologia de São Paulo, campus Bragança Paulista por seu apoio prático.

\section{Referências}

1 Flemings MC. Behavior of metal alloys in the semi-solid state. Metallurgical Transactions. A, Physical Metallurgy and Materials Science. 1991;22A:957-981.

2 Brown SB, Flemings MC. Net shape forming via semi-solid processing. Advanced Materials \& Processes. 1993;1:36-40.

3 Kapranos P, Ward PJ, Atkinson HV. Near net shaping by semi-solid metal processing. Materials \& Design. 2000;21:387-394.

4 Wang P, Cui JZ. Nondendritic microstructure of A356 alloy obtained by the nearby liquidus casting and thixoformed properties. Acta Metallurgica. 2002;38:952-955.

5 Atkinson HV. Modeling the semi-solid processing of metallic alloys. Progress in Materials Science. 2005;50:341-412.

6 Associação Brasileira do Alumínio. História do alumínio. São Paulo: ABAL; 2019 [acesso em 25 set. 2019 ]. Disponível em: http://abal.org.br/aluminio/historia-do-aluminio/

7 Infomet. Alumínio e suas ligas. Rio de Janeiro: Infomet; 2019 [acesso em 10 maio 2019]. Disponível em: http:// www.infomet.com.br/site/metais-e-ligas-conteudo-ler.php?codAssunto=108

8 Infomet. Ligas Al-Zn. Rio de Janeiro: INFOMET; 2019 [acesso em 10 maio 2019]. Disponível em: http://www. infomet.com.br/site/metais-e-ligas-conteudo-ler.php?codAssunto $=55$

9 Zoqui EJ, Lourençato LA, Benati DM. Thixoforming of aluminium-silicon alloys in a mechanical eccentric press. Diffusion and Defect Data, Solid State Data. Part B, Solid State Phenomena. 2008;141-143:517-522.

10 Torres LV. Avaliação da tixoconformabilidade das ligas AA7075 e AA7004 [dissertação]. Campinas: Universidade Estadual de Campinas; 2009.

11 Torres LV. Tixoconformação de novas ligas Al-Si-Cu [tese]. Campinas: Universidade Estadual de Campinas; 2013.

12 Torres, LV, Zoqui, EJ. Caracterização microestrutural das ligas AA7004 e AA7075 tixoforjadas em prensa excêntrica. Revista Matéria. 2019:e-12452.

13 Zoqui EJ, Torres LV. Evaluation of the thixoformability of AA7004 and AA7075 alloys. Materials Research. 2010;13:305-318.

14 Nadal RL, Roca AS, Fals HDC, Zoqui EJ. Mechanical properties of thixoformed hypoeutectic gray cast iron. Journal of Materials Processing Technology. 2015;226:146-156.

15 American Society for Testing and Materials. ASTM B557M-02a: standard test methods of tension testing wrought and cast aluminum and magnesium-alloy products [metric]. West Conshohocken: ASTM; 2002.

16 American Society for Testing and Materials. ASTM E112: standard test methods for determining average grain size. West Conshohocken: ASTM; 1996. 
17 Genda G, Yuyon C, Geing A. Mechanism of coarsening of dendrite during solidification. In: Solidification Processing: Proceedings of the Solidification Processing Conference; 1987 Sept; Sheffield; London. USA: N. American Publications Center; 1987. p. 416-419.

18 Kattamis TZ, Coughin JL, Flemings MC. Influence of coarsening on dendritic arm spacing of Al-Cu alloys. Transactions of the Metallurgical Society of AIME. 1967;239:1504-1511.

19 Callister WD, Rethwisch DG. Ciência e engenharia de materiais: uma introdução. 8. ed. Rio de Janeiro: LTC; 2012.

20 Chiarmetta G. Why thixo? In: S2P 2010: Proceedings of 6th International Conference on Semi-solid Processing of Alloys and Composites; 2000; Turin, Itália. Turin: Edimet; 2000. p. 15-21.

21 Torres LV, Zoqui EJ. Caracterização mecânica e metalúrgica das ligas tixoconformadas AA7004 e AA7075. Tecnologica em Metalurgia, Materiais e Mineração. 2019;16(3):407-413.

22 Vilas Bôas B. Avaliação do comportamento mecânico de ligas Al-Xwt\%Si-5wt\%Zn tixoconformadas [dissertação]. Campinas: Universidade Estadual de Campinas; 2012.

23 Chayong S, Atkinson HV, Kapranos P. Thixoforming 7075 aluminium alloys. Materials Science and Engineering A. 2005;390:3-12.

24 Jiang J, Atkinson HV, Wang Y. Microstructure and mechanical properties of 7005 aluminum alloy components formed by thixoforming. Journal of Materials Science and Technology. 2017;33:379-388.

Recebido em: 14 Out. 2019

Aceito em: 5 Abr. 2020 